\title{
DETERMINAÇÃO DE NITROGÊNIO AMONIACAL EM EFLUENTE LİQUIDO DA DIGESTÃO ANAERÓBIA DE MACRO E MICROALGAS
}

\author{
J. A. BORGES ${ }^{1}$, G. G. A. SILVA ${ }^{1}$, G. B. NUNES ${ }^{1}$, P. BERGOZZA ${ }^{1}$, J. A. V. $\operatorname{COSTA}^{1}$ e M. R. A. Z. \\ de SOUZA $^{1}$ \\ ${ }^{1}$ Universidade Federal do Rio Grande, Escola de Química e Alimentos \\ E-mail para contato: michrandrade@gmail.com
}

\begin{abstract}
RESUMO - O nitrogênio amoniacal presente em efluente da digestão anaeróbia, dependendo das concentrações, pode causar toxicidade e ser fator limitante do processo. Assim, sua determinação é uma importante análise de rotina em processos para a produção de biogás a partir da digestão anaeróbia de microalgas e macroalgas. Com o objetivo de comparar o desempenho do método de Nessler e o eletrodo íon seletivo na determinação da concentração do nitrogênio amoniacal em efluente de processo anaeróbio para produção de biogás, amostras de efluentes da digestão anaeróbia de biomassa da microalga Spirulina, biomassa de macroalgas e glicerol foram analisadas, utilizando como padrão solução de $\mathrm{NH}_{4} \mathrm{Cl}$. Ambos os métodos mostraram-se confiáveis. $\mathrm{O}$ eletrodo íon seletivo apresentou elevada linearidade da curva quando da análise de diferentes concentrações do padrão $\left(\mathrm{R}^{2}=0,999\right)$. Embora o método de Nessler forneça resultados confiáveis, tem a desvantagem de fazer uso de mercúrio, reagente tóxico. Seu pequeno intervalo de medição faz deste um método adequado para a análise de amostras com baixas concentrações de $\mathrm{N}-\mathrm{NH}_{4}{ }^{+}$, a exemplo dos efluentes de processos anaeróbios com biomassa de macroalgas. Por fornecer uma resposta rápida e direta, sem a necessidade de diluição da amostra, o eletrodo íon seletivo de amônia demonstrou ser o mais adequado para a determinação de $\mathrm{N}-\mathrm{NH}_{4}{ }^{+}$em efluentes de processos anaeróbios com biomassa de substratos com alto teor proteico, como a biomassa de microalgas.
\end{abstract}

\section{INTRODUÇÃO}

Devido a fatores como altas taxas de crescimento, as algas têm desempenhado papel de destaque no que concerne ao atual debate sobre as mudanças climáticas e a busca de alternativas energéticas frente ao uso dos combustíveis fósseis, atuando tanto na mitigação do dióxido de carbono atmosférico, como sendo matéria-prima para a produção de biodiesel, bioetanol e biogás. Estudos com digestão anaeróbia de biomassa algal são relatados desde a década de 1950 (Golueke et al., 1957), no entanto, a exploração mais acentuada desta biomassa com o intuito de obtenção de biogás teve início a partir do final da década de 1980, após a crise do petróleo, em que as macroalgas começaram a ser vistas como matéria-prima promissora para a produção biocombustíveis (Samson e LeDuy, 1986). Recentemente, a produção de biogás a partir de macroalgas e microalgas, e as 


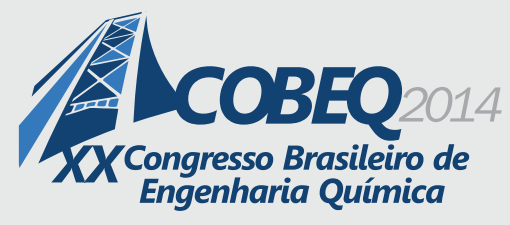

19 a 22 de outubro de 2014
Florianópolis/SC

condições operacionais que envolvem esses processos, têm sido objeto de crescente interesse pela comunidade científica (Vergara-Fernández et al., 2008; Tedesco et al., 2014; Jard et al., 2013).

No processo de digestão anaeróbia, as proteínas presentes no substrato são degradadas e convertidas em nitrogênio amoniacal, o qual pode encontrar-se sob as formas íon amônio $\left(\mathrm{NH}_{4}{ }^{+}\right)$ou amônia $\left(\mathrm{NH}_{3}\right)$, segundo o equilíbrio químico $\mathrm{NH}_{3}+\mathrm{H}^{+} \leftrightarrow \mathrm{NH}_{4}^{+}$. O nitrogênio amoniacal total (N$\mathrm{NH}_{4}{ }^{+}$) é uma fonte essencial de nitrogênio para os micro-organismos envolvidos no processo anaeróbio, no entanto, quando presente em altas concentrações, a $\mathrm{NH}_{3}$ pode representar toxicidade aos micro-organismos, e assim, causar inibição ao processo biológico para produção de biogás (Sheng et al., 2013; Liu e Sung, 2002). Por esse motivo, a determinação do $\mathrm{N}-\mathrm{NH}_{4}^{+}$em efluentes de processos anaeróbios da produção de biogás é uma análise de rotina importante, que pode contribuir para a garantia de melhores condições operacionais.

A concentração de $\mathrm{N}-\mathrm{NH}_{4}^{+}$em águas pode ser determinada por método titulométrico, colorimétrico e por eletrodo íon seletivo. Para concentrações superiores a $5 \mathrm{mg} . \mathrm{L}^{-1}$, utiliza-se o método titulométrico, em que a amostra é destilada, e após, a porção destilada é tratada com ácido bórico para ser titulada com solução de $\mathrm{H}_{2} \mathrm{SO}_{4}$. O término da titulação se dá quando há a mudança de coloração da solução para lilás. A concentração de $\mathrm{N}-\mathrm{NH}_{4}{ }^{+}$é diretamente proporcional ao volume de ácido gasto na titulação (Thompson e Morrison, 1951.). Entre os métodos colorimétricos estão o de fenol-hipoclorito, no qual fenol e hipoclorito reagem com $\mathrm{NH}_{4}{ }^{+}$formando um composto de coloração azul (Weatherburn, 1967), e o método de Nessler, que faz uso de reagente de mesmo nome, composto por potássio $(\mathrm{K})$, mercúrio $(\mathrm{Hg})$, e iodo $(\mathrm{I})$, que reagem com $\mathrm{NH}_{4}{ }^{+}$formando um composto coloidal de coloração castanho amarelado, que dependendo da intensidade apresentará uma determinada concentração de nitrogênio amoniacal (Nessler, 1856).

Dentre os métodos para esta análise, o de uso do eletrodo íon seletivo (EIS) é o operacionalmente mais simples. $\mathrm{O}$ equipamento consiste em um eletrodo de vidro alocado no interior de cilindro plástico, na parte inferior uma membrana permeável a gás separa a amostra da solução eletrolítica de referência de amônia contida no interior do cilindro. Após a adição de reagente alcalino à amostra (de forma que o $\mathrm{pH}$ da solução seja superior a 11 , no qual todo o $\mathrm{N}-\mathrm{NH}_{4}{ }^{+}$em solução está sob a forma de $\mathrm{NH}_{3}$ ), a $\mathrm{NH}_{3}$ se difunde através da membrana localizada na parte inferior do eletrodo, acarretando na alteração do potencial elétrico, sendo este medido para determinar a concentração de $\mathrm{N}-\mathrm{NH}_{4}{ }^{+}$(Arnold e Solsky, 1986).

Para a escolha de determinado método de análise alguns fatores são avaliados, tais como a redução de custos para análise, máxima precisão dos dados obtidos, mínima interferência do analista, menor consumo de reagentes e menor geração de resíduos (Merchert et al., 2012). Assim, o objetivo deste trabalho é comparar o desempenho do método de Nessler e o eletrodo íon seletivo na determinação da concentração do nitrogênio amoniacal em efluente de processo anaeróbio para produção de biogás.

\section{MATERIAL E MÉTODOS}

\subsection{Amostras}


As amostras analisadas foram provenientes de diferentes processos anaeróbios para produção de biogás a partir de biomassa de microalgas e macroalgas, como consta a seguir:

Amostra 1: Efluente de um biorreator anaeróbio alimentado com 10 g. $\mathrm{L}^{-1}$ de suspensão da microalga Spirulina (operado a $35^{\circ} \mathrm{C}$ );

Amostra 2: Efluente de um biorreator anaeróbio operando em co-digestão de $10 \mathrm{~g} \cdot \mathrm{L}^{-1}$ da microalga Spirulina e 5 g. $\mathrm{L}^{-1}$ de glicerol (operado a $35^{\circ} \mathrm{C}$ );

Amostra 3: Efluente de um biorreator anaeróbio operando em co-digestão de 5 g.L $\mathrm{L}^{-1}$ da microalga Spirulina +5 g. L $^{-1}$ de glicerol (operado a $35^{\circ} \mathrm{C}$ );

Amostra 4: Efluente de um biorreator anaeróbio alimentado com 7 g. $\mathrm{L}^{-1}$ de macroalgas (operado a 30 ${ }^{\circ} \mathrm{C}$ );

Amostra 5: Efluente de um biorreator anaeróbio alimentado com 10 g.L. $\mathrm{L}^{-1}$ de macroalgas (operado a $50{ }^{\circ} \mathrm{C}$ );

Amostra 6: Efluente de um biorreator anaeróbio operando em co-digestão de 3 g.L $\mathrm{L}^{-1}$ de microalgas + 4 g. $\mathrm{L}^{-1}$ de macroalgas (operado a $35^{\circ} \mathrm{C}$ );

Amostra 7: Efluente de um biorreator anaeróbio operando em co-digestão de 3 g.L $\mathrm{L}^{-1}$ de microalgas + 10 g. $\mathrm{L}^{-1}$ de macroalgas (operado a $35^{\circ} \mathrm{C}$ );

Amostra 8: Efluente de um biorreator anaeróbio alimentado com 10 g.L. $\mathrm{L}^{-1}$ de macroalgas (operado a $30{ }^{\circ} \mathrm{C}$ );

Amostra 9: Efluente de um biorreator anaeróbio alimentado com 4 g. $\mathrm{L}^{-1}$ de macroalgas (operado a 50 ${ }^{\circ} \mathrm{C}$ );

Amostra 10: Efluente de um biorreator anaeróbio alimentado com 7 g. $\mathrm{L}^{-1}$ de macroalgas (operado a $\left.50{ }^{\circ} \mathrm{C}\right)$.

Água destilada foi analisada como branco para ambos os métodos e solução de $\mathrm{NH}_{4} \mathrm{Cl}$ em concentrações de 1 a $20 \mathrm{mg} . \mathrm{L}^{-1}$ foi utilizada como padrão.

\subsection{Procedimentos Analíticos}

As amostras foram analisadas quanto à concentração de $\mathrm{N}-\mathrm{NH}_{4}{ }^{+}$pelo método de Nessler e por eletrodo íon seletivo, com e sem filtração em membrana de acetato de celulose com diâmetro de poro 0,45 $\mu \mathrm{m}$ (Merck Millipore, Brasil). Todas as análises foram realizadas em triplicata.

Método de Nessler: Para as análises pelo método de Nessler, $0,1 \mathrm{~mL}$ de reagente de Nessler (KI $+\mathrm{HgI}+\mathrm{NaOH}$ ) foi adicionado para cada $5 \mathrm{~mL}$ de amostra de efluente em tubos de ensaio. Após, as amostras foram deixadas em repouso e sob a proteção da luz por $10 \mathrm{~min}$. As concentrações de N$\mathrm{NH}_{4}{ }^{+}$foram determinadas a partir da leitura em espectrofotômetro (Quimis Q798DRM, Brasil) a 525 $\mathrm{nm}$, com curva padrão de $\mathrm{NH}_{4} \mathrm{Cl}$ em concentrações de 0 a $20 \mathrm{mg} \cdot \mathrm{L}^{-1}$ de $\mathrm{N}_{-} \mathrm{NH}_{4}{ }^{+}$.

Método do eletrodo íon seletivo: A determinação de $\mathrm{N}_{-} \mathrm{NH}_{4}{ }^{+}$foi realizada com eletrodo íon seletivo (HANNA HI 4101, EUA) composto por eletrodo de referência de pH interno, alocado no 
interior de recipiente termoplástico e isolado da amostra por membrana de politetrafluoretileno permeável a gás. Para a análise, 1 mL da solução ISA (HANNA HI 4001-00, EUA) (solução alcalina, para ajuste de $\mathrm{pH}$ ) foi adicionado a $50 \mathrm{~mL}$ de amostra contida em recipiente de polietileno. Imediatamente após a adição da solução alcalina, o recipiente contendo a amostra foi fechado com tampa na qual estava acoplada o eletrodo íon seletivo, para evitar a perda de gás. A leitura foi realizada em mg. $\mathrm{L}^{-1}$ de $\mathrm{N}-\mathrm{NH}_{3}$.

\subsection{Análise Estatística}

Os resultados médios foram avaliados por Análise de Variância e Teste de Tukey para determinar as possíveis diferenças entre as médias, utilizando nível de confiança de $95 \%$.

Os indicativos de desempenho utilizados foram os limites de detecção e quantificação, e a precisão (repetitividade), com seis repetições de análise. O cálculo do limite de detecção (LD) foi realizado com base na Equação 1, em que a mínima concentração do analito presente na amostra relaciona a média ( $\bar{X}$ ) e os desvios padrão $(s)$ do branco, e valor de " $t$ " da distribuição de Student para o número de graus de liberdade $(g l)$ estudado a $95 \%$ de confiança $(1-\alpha=0,05)$.

$$
L D=\bar{X}+t_{(g l ; 1-\alpha)} * s
$$

O limite de quantificação (LQ) foi calculado a partir da Equação 2, a partir dos valores de limite de detecção (LD).

$$
L Q=3 * L D
$$

A precisão (repetitividade) dos dados foi avaliada conforme o coeficiente de variação (CV), calculado segundo a Equação 3.

$$
C V(\%)=\frac{s}{\bar{X}} * 100
$$

\section{RESULTADOS E DISCUSSÃO}

A Tabela 1 apresenta as características dos métodos estudados para determinação da concentração de $\mathrm{N}-\mathrm{NH}_{4}{ }^{+}$. Devido ao seu maior intervalo de calibração, a linearidade da curva de medição da solução padrão de $\mathrm{NH}_{4} \mathrm{Cl}$, para o eletrodo íon seletivo, manteve-se constante até a concentração de $100 \mathrm{mg} \cdot \mathrm{L}^{-1}$ de $\mathrm{NH}_{4}{ }^{+}$(maior concentração analisada), com coeficiente de correlação $\left(\mathrm{R}^{2}\right)$ de $0,9990 \pm 0,0011$. Tal fato incorre em vantagem do uso do eletrodo íon seletivo frente ao método de Nessler, não havendo a necessidade de diluições das amostras que apresentem concentrações superiores a $20 \mathrm{mg} \cdot \mathrm{L}^{-1}$ de $\mathrm{NH}_{4}{ }^{+}$, concentração após a qual a curva de calibração da 
solução padrão pelo método de Nessler deixa de ser linear.

Tabela 1 - Características avaliadas dos métodos para determinação da concentração de $\mathrm{N}-\mathrm{NH}_{4}{ }^{+}$.

\begin{tabular}{|c|c|c|}
\hline & Método de Nessler & Eletrodo íon seletivo \\
\hline Medida do sinal analítico & Espectrofotômetro & Potencial elétrico \\
\hline Reagente utilizado para análise & Nessler $(\mathrm{KI}+\mathrm{HgI}+\mathrm{NaOH})$ & $\mathrm{NaOH}$ \\
\hline Volume de amostra $(\mathrm{mL})$ & 5 & 50 \\
\hline Intervalo de medição $\left(\mathrm{mg} . \mathrm{L}^{-1} \mathrm{NH}_{4}^{+}\right)$ & $1-20$ & $1-1000$ \\
\hline Limite de detecção $\left(\mathrm{mg} . \mathrm{L}^{-1} \mathrm{NH}_{4}^{+}\right)$ & 0,23 & 0,30 \\
\hline Limite de quantificação $\left(\mathrm{mg}^{+} \mathrm{L}^{-1} \mathrm{NH}_{4}^{+}\right)$ & 0,68 & 0,89 \\
\hline $\mathrm{CV}(\%)$ & 2,1 & 1,8 \\
\hline
\end{tabular}

Os valores de limite de detecção e quantificação para o método de Nessler foram inferiores aos encontrados para o método com eletrodo íon seletivo. Segundo a IUPAC (siga em inglês para União Internacional de Química Pura e Aplicada) (Thompson et al., 2002), o limite de detecção é a menor quantidade do analito que pode ser medida com confiabilidade estatística razoável, enquanto o limite de quantificação corresponde à concentração abaixo da qual o método analítico não pode operar com uma precisão aceitável. Para ambos os métodos estudados, os limites de detecção e quantificação ficaram abaixo da mais baixa concentração do padrão analisada $\left(1 \mathrm{mg} \cdot \mathrm{L}^{-1}\right)$.

A Figura 1 apresenta as concentração de $\mathrm{N}_{-} \mathrm{NH}_{4}{ }^{+}$para as amostras analisadas, em que percebese que aquelas provenientes de processos anaeróbios utilizando a biomassa da microalga Spirulina como substrato apresentaram as maiores concentrações de nitrogênio amoniacal. Tal fato ocorre devido o alto teor proteico da biomassa dessa microalga (Morais et al., 2009). Das 10 amostras de efluentes analisadas com microfiltração, para a amostra 5 não houve diferença significativa $(p>0,05)$ entre os resultados obtidos por ambos os métodos. A amostra 4 apresentou maiores resultados de concentração de $\mathrm{N}-\mathrm{NH}_{4}{ }^{+}$quando analisada pelo método de Nessler, enquanto as demais $(1,2,3,6$, e 7) apresentaram os maiores resultados de $\mathrm{N}_{-} \mathrm{NH}_{4}{ }^{+}$quando analisadas por eletrodo íon seletivo. Para estas últimas, as concentrações de $\mathrm{N}_{-} \mathrm{NH}_{4}{ }^{+}$eram superiores a $200 \mathrm{mg} . \mathrm{L}^{-1}$. Essa diferença nos resultados obtidos para as amostras analisadas pelo método de Nessler e por eletrodo íon seletivo quando presente em maiores concentrações de $\mathrm{N}_{-} \mathrm{NH}_{4}{ }^{+}$é devido à faixa de concentração para a qual o método de Nessler é mais adequado $\left(1-20 \mathrm{mg} . \mathrm{L}^{-1}\right.$ de $\left.\mathrm{N}-\mathrm{NH}_{4}{ }^{+}\right)$. Efluentes provenientes da digestão anaeróbia de microalgas com alto teor de proteínas, como é caso da microalga Spirulina, costumam ter concentrações de nitrogênio amoniacal igual ou superiores a essa (Borges et al., 2013). Devido a isso, para a análise de efluentes da digestão anaeróbia de microalgas, o eletrodo íon seletivo de amônia é o mais adequado, quando comparado ao método de Nessler. Diferentemente do que ocorre para as amostras provenientes da digestão anaeróbia de macroalgas, as quais costumam ter menores concentrações de nitrogênio amoniacal (Franck, 2012).

No entanto, quando observadas as amostras que não foram filtradas (amostras 8, 9 e 10), essa relação da concentração de $\mathrm{N}-\mathrm{NH}_{4}{ }^{+}$da amostra com o método mais adequado a ser utilizado, não foi verificada da mesma forma, visto que as três amostras eram provenientes de processos anaeróbios 
com biomassa de macroalgas, e portanto, com baixas concentrações de $\mathrm{N}_{-} \mathrm{NH}_{4}{ }^{+}$. Não houve diferença significativa $(p>0,05)$ entre os métodos analisados para a amostra 8 , enquanto para a amostra 9 a concentração de $\mathrm{N}^{-\mathrm{NH}_{4}}{ }^{+}$encontrada pelo método de Nessler foi superior ao eletrodo íon seletivo, e o contrário foi observado para a amostra 10. Essa diferença entre os dois métodos quando comparadas as amostras sem microfiltração pode ser devido à presença de interferentes nas amostras que não receberam este tratamento antes de analisadas. Alguns autores relatam que a presença de alguns interferentes prejudicam os resultados obtidos por ambos os métodos. Jeong et al., 2013, ao estudarem o método de Nessler com e sem modificação para a análise de $\mathrm{N}_{-} \mathrm{NH}_{4}{ }^{+}$em água, verificaram que a presença de $\mathrm{Fe}^{2+}$ em concentrações superiores a $1 \mathrm{mg} . \mathrm{L}^{-1}$, influencia nos resultados, não sendo obtidos resultados confiáveis, pois o $\mathrm{Fe}^{2+}$ é capaz de formar complexos com os sais do reagente de Nessler aumentando a turbidez das amostras analisadas. Por outro lado, Molins-Legua et al. (2006), ao comparar diferentes métodos para determinação de amônia em águas, constataram que em amostras contendo aminas e compostos aromáticos nitrogenados, estes atuam como interferentes na análise por eletrodo íon seletivo.

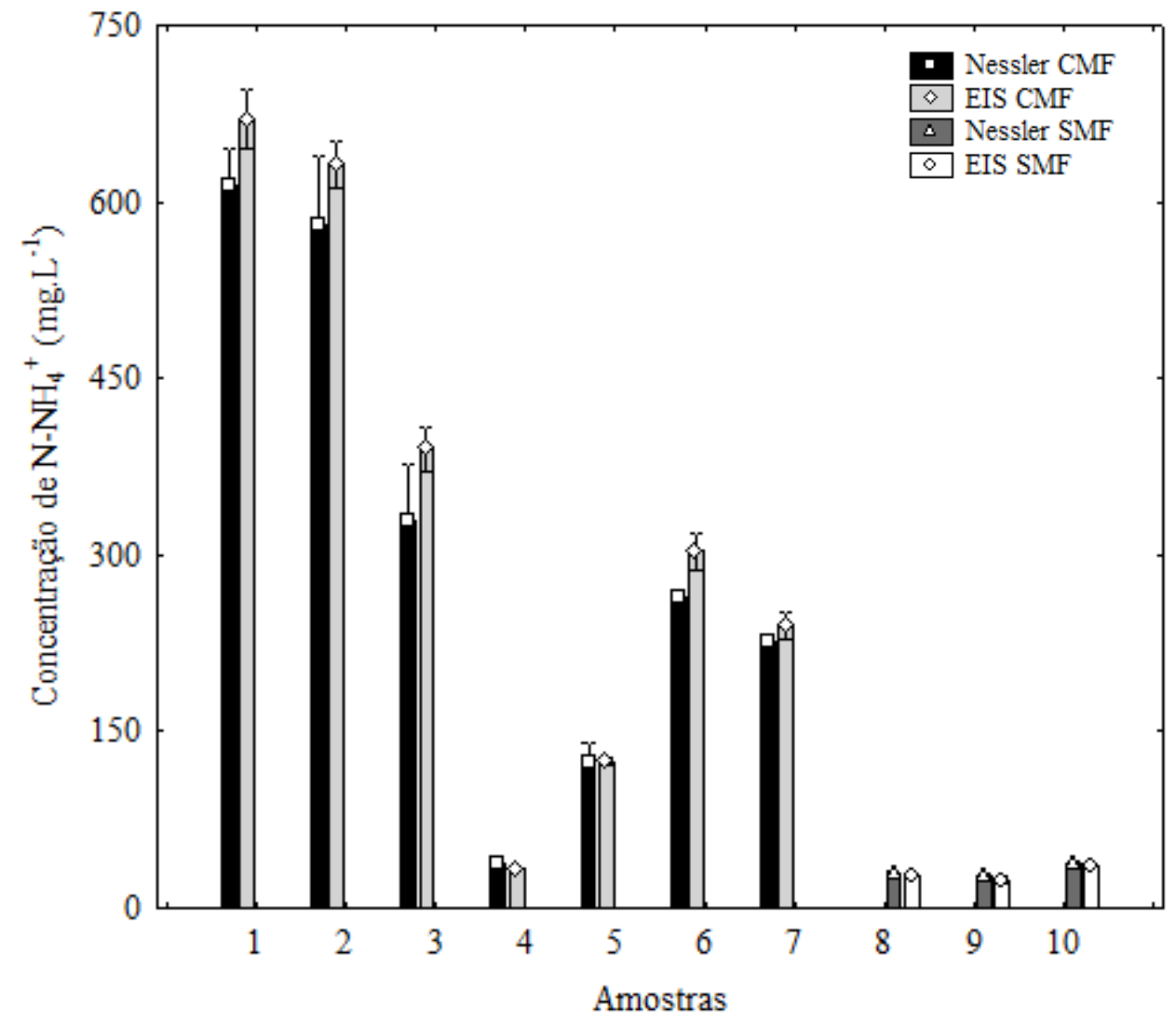

Figura 1 - Concentração de $\mathrm{N}_{-} \mathrm{NH}_{4}{ }^{+}$nas amostras de efluentes da digestão anaeróbia analisadas pelo método de Nessler e eletrodo íon seletivo (EIS), com (CMF) e sem microfiltração (SMF).

\section{CONCLUSÕES}

O presente trabalho comparou os resultados de determinação de $\mathrm{N}_{-} \mathrm{NH}_{4}{ }^{+}$a partir do método de 


\section{9 a 22 de outubro de 2014 \\ Florianópolis/SC}

Nessler e eletrodo íon seletivo. Ambos os métodos demonstraram ser confiáveis para a determinação de N-NH${ }_{4}{ }^{+}$, apresentando baixos limites de detecção $\left(<0,3 \mathrm{mg} \cdot \mathrm{L}^{-1}\right)$ e quantificação $\left(<0,9 \mathrm{mg} \cdot \mathrm{L}^{-1}\right)$, e coeficientes de variação $<3 \%$. Embora a análise com reagente de Nessler forneça resultados confiáveis, fazendo uso de menor volume de amostra quando comparado ao eletrodo íon seletivo, deve-se levar em consideração o fato deste método empregar mercúrio, reagente tóxico e, além disso, a reação do reagente com o N-NH${ }_{4}{ }^{+}$forma um composto coloidal, sendo este passível de floculação e o seu grau de dispersão importante na intensidade da cor final da solução.

A determinação de $\mathrm{N}^{-\mathrm{NH}_{4}}{ }^{+}$nos efluentes provenientes da digestão anaeróbia de macroalgas, devido às suas menores concentrações $\left(24-124 \mathrm{mg} \cdot \mathrm{L}^{-1}\right)$, demonstraram ser mais adequadamente analisadas segundo o método de Nessler. No entanto, o emprego do método de Nessler demonstrou não ser o mais confiável para a quantificação de $\mathrm{N} \mathrm{NH}_{4}{ }^{+}$em efluentes da digestão anaeróbia de microalgas com alto teor proteico, devido às elevadas concentrações de $\mathrm{N}^{-\mathrm{NH}_{4}}{ }^{+}$encontradas $(227-$ $670 \mathrm{mg} . \mathrm{L}^{-1}$ ), sendo o eletrodo íon seletivo o mais adequado neste caso. A análise com eletrodo íon seletivo tem a vantagem de fornecer uma resposta rápida e direta, sem a necessidade de diluição da amostra, além de ser metodologia analítica limpa, sem geração de resíduos tóxicos, evitando assim, possíveis contaminações do analista e do ambiente quando do descarte do resíduo.

Através dos resultados apresentados foi possível determinar, o método mais adequado, dentre os estudados, para a quantificação de $\mathrm{N}_{-} \mathrm{NH}_{4}{ }^{+}$em efluentes de processos anaeróbios envolvendo a biomassa de microalgas e macroalgas, que se faz relevante devido o crescente interesse em estudos para a produção de biogás a partir de biomassa algal, e a importância que tal determinação tem para o controle das condições ideais deste processo.

\section{AGRADECIMENTOS}

Os autores agradecem a CAPES (Coordenação de Aperfeiçoamento de Pessoal de Nível Superior) e ao PDE/FURG (Programa de Desenvolvimento do Estudante da Universidade Federal do Rio Grande) pelo apoio financeiro para a realização deste trabalho.

\section{REFERÊNCIAS}

ARNOLD, M. A.; SOLSKY, R. L. Ion-Selective Electrodes. Anal. Chem., v. 58, p. 84R-101R, 1986.

BORGES, J. A.; ROSA, G. M.; MEZA, L. H. R.; HENRARD, A. A.; SOUZA, M. R. A. Z., COSTA, J. A. V. Spirulina sp. LEB-18 culture using effluent from the anaerobic digestion. Braz. J. Chem. Eng., v. 30, n. 2, p. 277-287, 2013.

FRANCK, D. T. Produção de biogás a partir de macroalgas. Mestrado em Engenharia e Ciência de Alimentos - Universidade Federal do Rio Grande (FURG), 2012.

GOLUEKE, G.; OSWALD, W. J.; GOTAAS, H. B.. Anaerobic digestion of algae. Appl Microbiol., v. 5, n. 1, p. 47-55, 1957. 
JARD, G.; DUMAS, C.; DELGENES, J. P.; MARFAING, H.; SIALVE, B.; STEYER, J. P.; CARRÈRE, H. Effect of thermochemical pretreatment on the solubilization and anaerobic biodegradability of the red macroalga Palmaria palmata. Biochem. Eng. J., v. 79, p. 253- 258, 2013.

JEONG, H.; PARK, J.; KIM, H. Determination of $\mathrm{NH}_{4}{ }^{+}$in Environmental Water with Interfering Substances Using the Modified Nessler Method. J. Chem., v. 2013, p. 1-9, 2013.

LIU, T.; SUNG, S. Ammonia inhibition on thermophilic aceticlastic methanogens. Water Sci. Technol., v. 45, n. 10, p. 113-120, 2002.

MELCHERT, W. R.; REIS, B. F.; ROCHA, F. R. P. Green chemistry and the evolution of flow analysis: A review. Anal. Chim. Acta, v. 714, p. 8-19, 2012.

MOLINS-LEGUA， C.; MESEGUER-LLORET, S.; MOLINER-MARTINEZ, Y.; CAMPÍNSFALCO, P. A guide for selecting the most appropriate method for ammonium determination in water analysis. Trends Anal. Chem., v. 25, p. 282-290, 2006.

MORAIS, M. G.; RADMANN, E. M.; ANDRADE, M. R.; TEIXEIRA, G. G.; BRUSCH, L. R. F.; COSTA, J. A. V. Pilot scale semicontinuous production of Spirulina biomass in southern Brazil. Aquaculture, v. 294, p. 60-64, 2009.

NESSLER, J. A. Colorimetric determination of ammonia by Nessler reagent. Chemisches Zentralblatt, v. 27, p. 529-541, 1856.

NIEDZIELSKI, P.; KURZYCA, I.; SIEPAK, J. A new tool for inorganic nitrogen speciation study: Simultaneous determination of ammonium ion, nitrite and nitrate by ion chromatography with postcolumn ammonium derivatization by Nessler reagent and diode-array detection in rain water samples. Anal. Chim. Acta., v. 577, p. 220-224, 2009.

SAMSON, R. e LEDUY, A. Detailed study of anaerobic digestion of Spirulina maxima algal biomass. Biotech. Bioeng., v. 28, p. 1014-1023, 1986.

SHENG, K.; CHEN, X.; PAN,J.; KLOSS, R.; WEI, Y.; YING, Y. Effect of ammonia and nitrate on biogas production from food waste via anaerobic digestion. Biosyst. Eng., v. 116, p. 205-212, 2013.

TEDESCO, S.; BENYOUNIS, K. Y.; OLABI, A. G. Mechanical pretreatment effects on macroalgaederived biogas production in co-digestion with sludge in Ireland. Energy, v. 61, p. 27-33, 2013.

THOMPSON, M.; ELLISON, S. L. R.; WOOD, R. Harmonized guidelines for single-laboratory validation of methods of analysis (IUPAC Technical Report). Pure Appl. Chem., v. 74, p. 835-855, 2002.

THOMPSON, J. F.; MORRISON, G. R. Determination of organic nitrogen: Control of variables in the use of Nessler's reagent. Anal. Chem., v. 23, n. 8, p. 1153-1157, 1951.

VERGARA-FERNÁNDEZ, A.; VARGAS, G.; ALARCÓN, N.; VELASCO, A. Evaluation of marine algae as a source of biogas in a two-stage anaerobic reactor system. Biomass Bioenergy, v. 32, p. 338344, 2008.

WEATHERBURN, M. W. Phenol-hypochlorite reaction for determination of ammonia. Anal. Chem., v. 39, n. 8, p. 971-974, 1967. 